\title{
Ultraviolet radiation detector to obtain the energy and rate of particles at different heights
}

\section{E. Ponce ${ }^{* \dagger}$}

Autonomous University of Puebla, Mexico.

E-mail: eponceefcfm.buap.mx

\begin{abstract}
The nature and origin of cosmic rays remains one of the greatest puzzles of modern astrophysics after more than 50 years since their first registration. Several ground experiments have reported the rate registered at its height of operation. To continue with the study of cosmic rays, it is proposed obtain the rate at different heights in the Earth's atmosphere, developing a small and portable ultraviolet radiation detector, consisting of a scintillation plastic, a PMT, and a fast DAQ system. In this work, I present the design and construction of the UV detector, the events and rate recorded in Pico de Orizaba national park, campus Autonomous University of Puebla-Mexico, at a height of 4200 meters above sea level approximately. The rate obtained will compared with the number of registered particles per second, provided by experiments operating at heights known.
\end{abstract}

35th International Cosmic Ray Conference - ICRC2017

10-20 July, 2017

Bexco, Busan, Korea

* Speaker.

${ }^{\dagger}$ CONACyT, Mexico city, Mexico, VIEP-BUAP, Puebla, Mexico. 


\section{Introduction}

The Earth's atmosphere is subject to constant arrival of atomic nuclei, mostly protons, known as primary cosmic radiation; during it's trajectory to the surface, by interacting with atmospheric nuclei, produce a variety of elementary particles, identified as secondary cosmic rays. In order to obtain the rate of cosmic particles, I design a portable ultraviolet radiation detector, which consists of a small scintillator plastic, thick $1 \mathrm{~cm}$, radius $2 \mathrm{~cm}$, threshold energy approximately one $\mathrm{MeV}$; how the interaction of high energy cosmic rays with scintillation plastic molecules, produces photons, this gives the opportunity for the light is detected using a photomultiplier tube (PMT) the PMT converts photons into an electrical signal, finaly, it is processed by a DAQ system of high speed. In this paper, we present the portable ultraviolet radiation detector, and the laboratory calibration, the next step will be to test the detector performance, operating during the journey from university city of BUAP campus (2200 meters above sea level) to Pico de Orizaba national park (4200 meters above sea level) in Mexico. The data obtained will be compared with the rate registered by experiments operating at heights known as AUGER, HAWC, LAGO.

\section{Ultraviolet radiation detector setup}

To obtain the continuous rate of the cosmic rays at different heights, it is necessary a detector operating for each specific height; for this reason I developed a small ultraviolet radiation detector the main purpose of the experiment is obtain the cosmic rays rate of experimental form in the range of heights of 2200 to 4200 meters above sea level, approximately.

The UV detector [1] consists of a very small scintillator plastic as first transducer, see figure 1. Scintillation detectors make use of special materials, when exposed to ionizing radiation, they emit a small flash of light. This phenomenon occurs when radiation interacts with matter, exciting and ionizing one large number of atoms and molecules, when they return to their steady state, emit photons in the visible spectrum or around it.
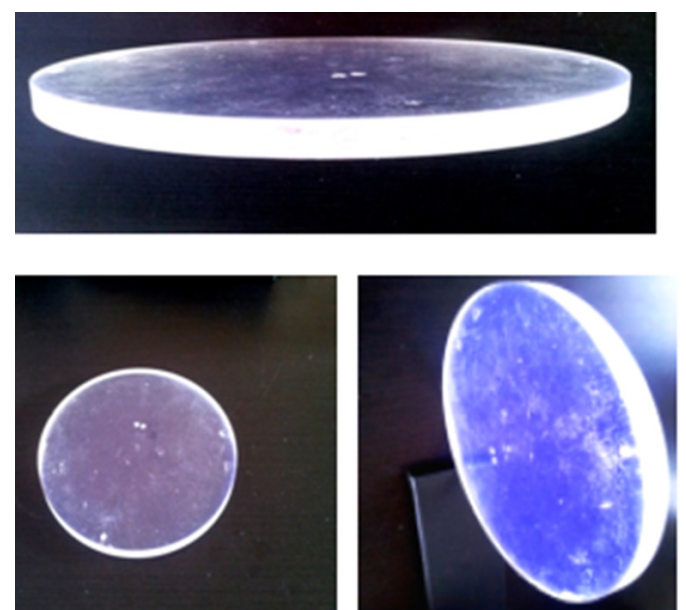

Figure 1: Scintillation plastic as a medium radiator, thick $1 \mathrm{~cm}$, radius $2 \mathrm{~cm}$, threshold energy $\sim 1 \mathrm{MeV}$. Used in the UV radiation detector to obtain the rate of particles at different heights. 
To obtain useful information from scintillation plastic, a photodetector is added [2], which converts the photons into an electrical signal. The photodetector most frequently used for any scintillator detector is a photomultiplier tube (PMT), this device, has a photocathode, an electron multiplier chamber and a polarization base as main parts; the first part of the PMT is a thin layer of a special material that emits electrons (more know as photoelectrons) when photons pass through the photocathode. The second part of the PMT, is an array of electrodes polarized with an active (semiconductor devices) or passive (resistive) base, to keep a gradual distribution of voltage in each electrode and achieve gains of the order up to $10^{6}$, resulting a current pulse in the PMT output pin; connecting a load resistor in the output, a voltage is generated in the order of a few millivolts which is processed by a DAQ system for further analysis, in order to obtain information about the incident radiation. In figure 2 shows all the necessary parts, only need to connect five volts and an SPI interface to control the correct PMT's operation.

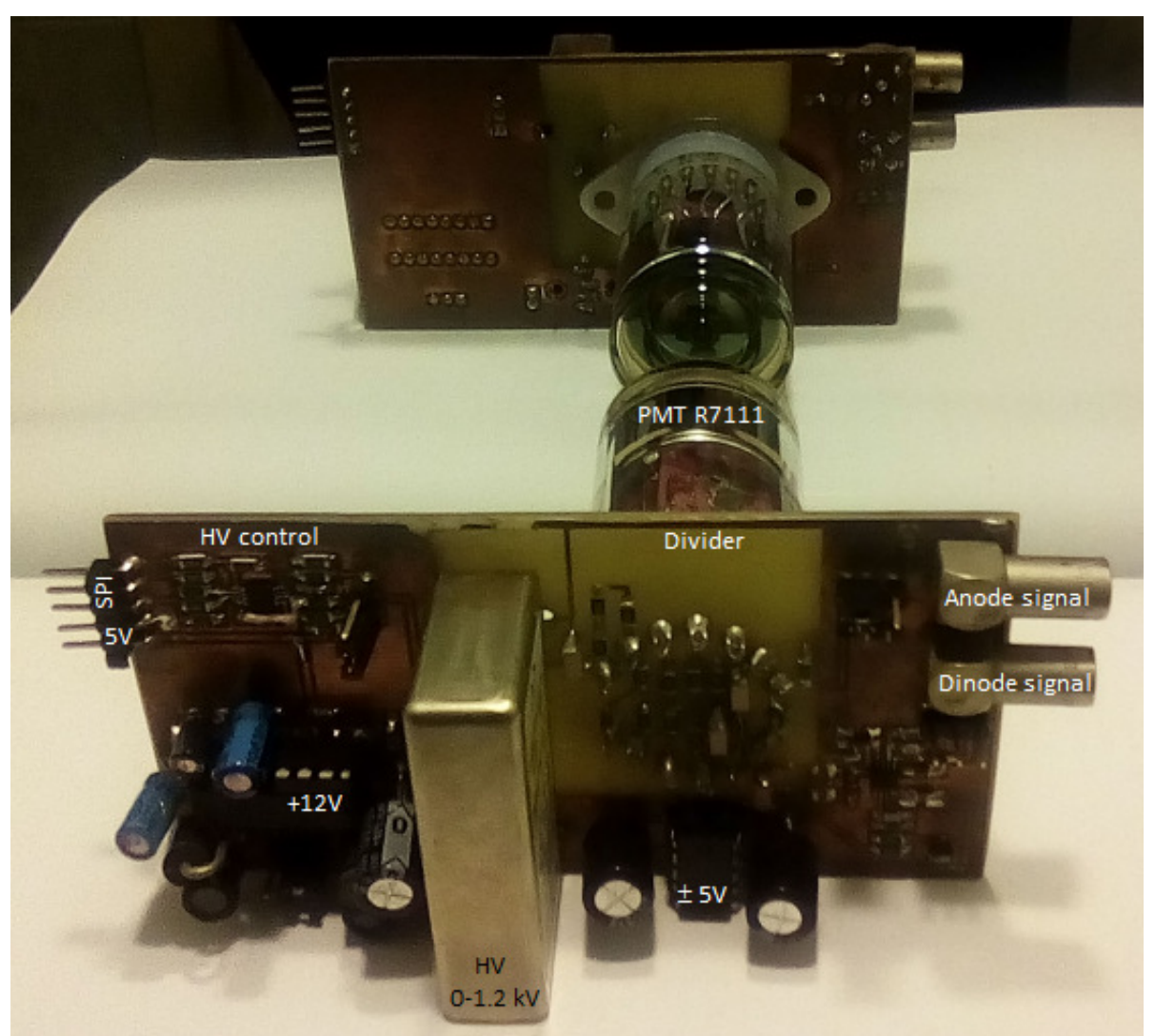

Figure 2: Portable UV detector contains one R7111 photomultiplier tube from the Hamamatsu company.

The DAQ system has as main computer an FPGA Xilinx, as shown in figure 3, this controls the information of a digitalization board every $10 \mathrm{~ns}$; a GPS and a temperature-pressure sensor every second; a high voltage source to control the constant gain of the photomultiplier [3], every minute. The time intervals assigned, are with the purposes of autocalibrate the UV detector during the trajectory of operation in the journey. 


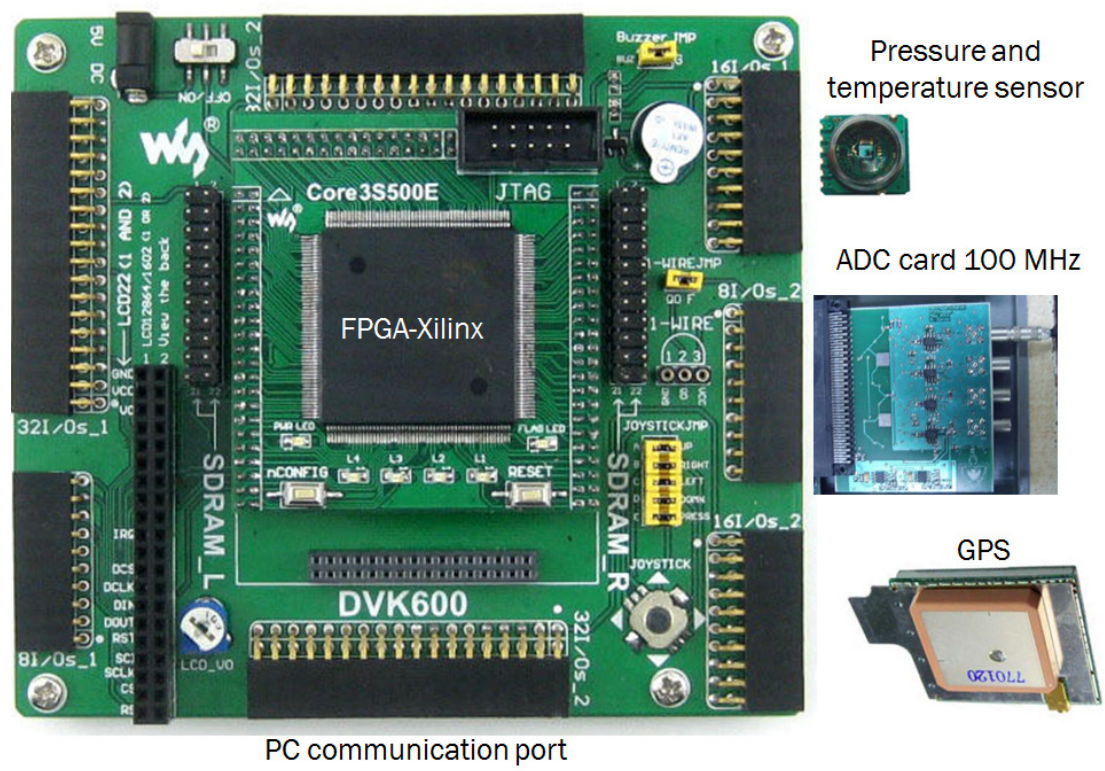

Figure 3: DAQ system of high speed processing, has as main computer an FPGA Spartan 3S500 Xilinx family, processes the data of a temperature and pressure sensor HP03S, a fast digitalization board series BUAP-R.Conde, a GPS GY-GPS6MV2, the HV serial port interface and the communication port with the PC.

\section{Ultraviolet radiation detector testing and preliminary results}

As a first step in the calibration process of the UV detector, we measured the PMT's response to a single photoelectron signal (SPE) [4] figure 3. From these measurements we obtained the signal shape for a window time of $5 \mathrm{~ms}$; figure 5 shows the fist $512 \mathrm{~ns}$. The rate recorded each five millisecons shows in figure 6.

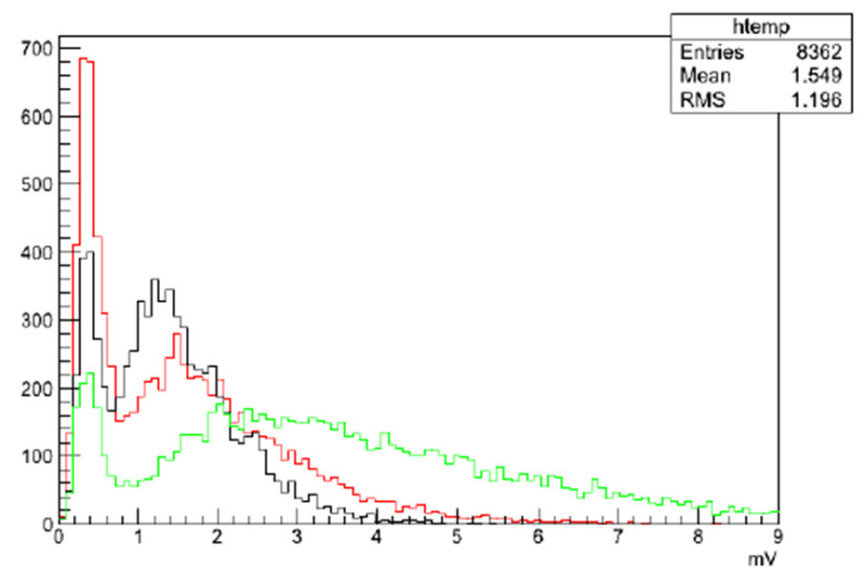

Figure 4: Single photoelectron response of photomultiplier R7111, for different voltages: $0.850 \mathrm{kV}$ (green signal), $0.900 \mathrm{kV}$ (red signal) and $1.0 \mathrm{kV}$ (black signal). 


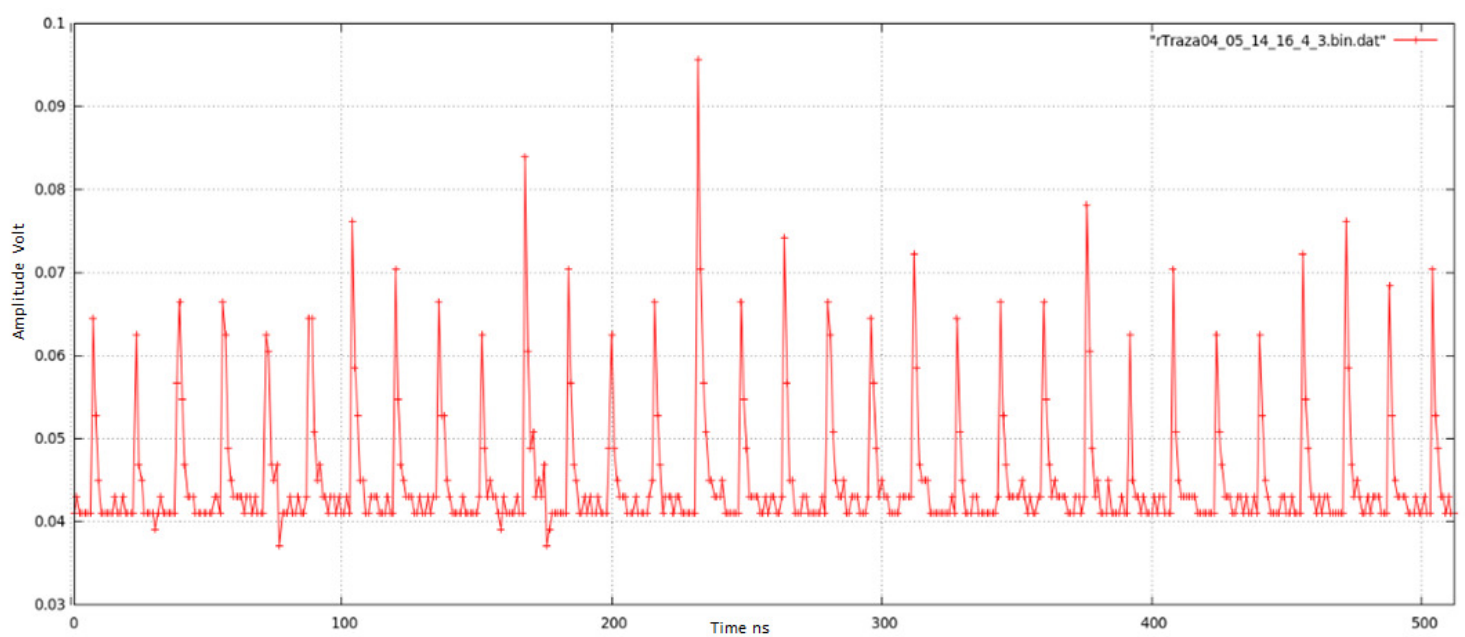

Figure 5: Traces recorded in Pico de Orizaba National Park at a height of 4200 meters above sea level Puebla-Mexico.

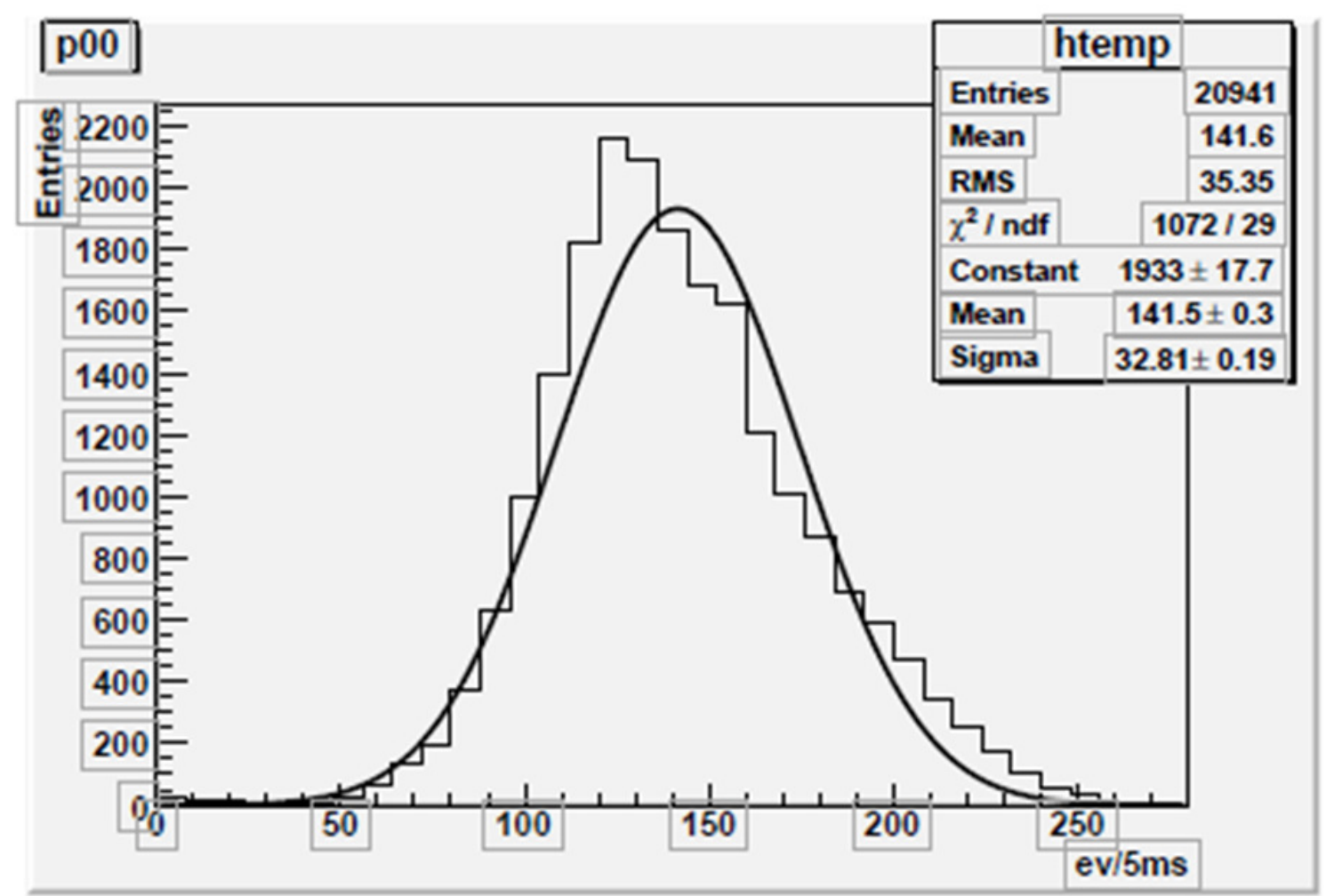

Figure 6: Histogram of particles, the maximun peak is around 140 each $5 \mathrm{~ms}$, in one second the histogram will increase 200 times, with this consideration the rate is around $28 \times 10^{3}$ particles/second. 


\section{Conclusions}

In this paper, was presented the portable ultraviolet radiation detector, together with results obtained at a height of 4200 meters above sea level. The next step will be to test the portable ultraviolet detector performance, in Puebla-Mexico, operating during the journey from university city of BUAP campus to Pico de Orizaba national park. The data obtained were compared with the number of registered particles per second, provided by experiments operating at heights known as AUGER, LAGO, etc. In the near future will create a database with free access, recording the profile signal processed by DAQ system, generated by each particle, for further and off line analysis, by students or researchers interested.

\section{References}

[1] Dmitriev, UV radiation from the night-time atmosphere seen from the Universitetsky-TATIANA satellite, in proceedings of AIP $\mathbf{1 1 1 8 .}$

[2] Garipov G.K., Program of transient UV event research at TATIANA-2 satellite, Journal of Geophysical Research A 115.

[3] E. Ponce., Pinhole camera for study of atmospheric UV flashes as a source of background in the TUS experiment), Nuclear Instruments and Methods in physics research A 639.

[4] E. Ponce., Transient Luminous events registered with a ground pinhole), in proceedings of The 34th International Cosmic Ray Conference, PoS (ICRC2015) 437. 